\title{
M(t)/M/1 Queueing System with Sinusoidal Arrival Rate
}

\author{
A.P. Pant ${ }^{1}$, R.P. Ghimire ${ }^{2}$ \\ ${ }^{I}$ Department of Mathematics, Tri-Chandra Campus, Ghantaghar, TU, Kathmandu, Nepal \\ ${ }^{2}$ Department of Natural Sciences (Mathematics), Kathmandu University, Kavre, Nepal \\ Corresponding author: pantananda88@gmail.com
}

\begin{abstract}
This paper deals with the study of $M(t) / M / 1$ queueing system with customers arrive to the system with sinusoidal arrival rate function $\lambda(t)$ and are served exponentially with the rate $\mu$. On formulating the mathematical model, we obtain the expressions for mean waiting time in the queue, mean time spent in the system, mean number of customers in the queue and in the system by using recursive method. Some numerical illustrations are also obtained by using computing software so as to show the applicability of the model under study.
\end{abstract}

Keywords: Queue, Sinusoidal function, Exponential, Probability

\section{Introduction}

From very beginning of introduction of queueing system, several authors studied M/M/1 queueing model in various provisions and frameworks. Of course the $\mathrm{M} / \mathrm{M} / 1$ model has been studied extensively and much is known about its time - dependent or transient behavior. Some of the pioneering and latest works are notable. Abate and Whitt [1] described the evolution of the classical M/M/1 queue. They obtained simple approximations and structural theorems that expose the essential nature of the transient behavior. Abate and Whitt [2] developed a better understanding of the transient behavior of queues and related stochastic flow systems so that we can provide relatively simple descriptions suitable for engineering purposes. Baccelli and Massey [3] presented a derivation of the transient distribution for the queue length and the busy period of the $\mathrm{M} / \mathrm{M} / 1$ queue that follows purely from the sample path behavior of the process. Li et al. [16] studied M/M/1 queues in which the service rates depend on the number of the customers served since the beginning of the current busy period. They presented a simple and computationally tractable scheme which recursively computes the stationary probabilities of the queue length. Kinateder and Lee [15] provided a new approach to the computation of the Laplace transform of the length of the busy period of the M/M/1 queue with constrained workload (finite dam) without the use of complex analysis. Choudhury and Borthakur [4] dealt with statistical inference with regard to the classical single server Markovian queueing model. Ghimire and Ghimire [7] dealt with the study of M/M/1 queue with heterogeneous arrival and departure with the provision of server vacations and breakdowns customer arrive service facilities with poison process and exponential service time distribution. Murthy et al. [19] studied a generalized queueing model in 
which customers are served as a batch of size $\mathrm{k}$ at a time except when there are less than $\mathrm{k}$ customers in the system at the time of service. For developing these independent models with bulk service rule, they made use of the dependence structure given by Rao K.S (1986). Maurya [17] explored the probability generating functions using Rouche's theorem in both cases of slower and faster arrival rates of the queueing model into consideration. Jindal and Sharma [13] studied M/M/1 queue under non preemptive service priority discipline. Rasouli et al. [20] proposed a new analytical model to estimate the energy consumption in clustered WSNS using $\mathrm{M} / \mathrm{M} / 1$ queueing model for all sensor nodes and developed an analytical model for energy saving by reducing the number of transitions between idle state and active state in all sensor nodes. Recently, Kalidass and Ramanath [14] explained explicit expressions for the time dependent probabilities of the $\mathrm{M} / \mathrm{M} / 1$ queue with server vacations under a multiple vacation scheme. They also obtained the dependent performance measures of the system. Ibe and Isijola [11] dealt with an $\mathrm{M} / \mathrm{M} / 1$ queueing system in which two types of vacations can be taken by the server. Maurya [18] demonstrated a mathematical modeling for analyzing a Markovian queueing system with two heterogeneous servers and working vacation and obtained various performance measures of the Markovian queueing system with varying parameters under steady state using matrix geometric method.

M/M/1 queueing model studied by aforementioned several authors could not address some real queueing problems where in arrival rate function is piecewise continuous and occurs in real life situations in international border checking process where arrival rate function is characterized by sinusoidal function $\lambda(\mathrm{t})=\square+\beta \sin (\gamma \mathrm{t})$ in the case when arrival rate rapidly fluctuates, where $\propto$ is the delay probability and $\beta$ is the service quality parameter that $\beta=\square \propto$ for positive constants $\square, 0<\alpha<1$ and $\gamma=2 \pi / \Psi, \Psi$ is the cycle length or period.Sinusoidal arrival rate function is applicable when we impose the flow control policy in which customer is expected to arrive within an agreed appointment time window instead of at a specific appointment time. Very rare literatures can be found in which the sinusoidal arrival rate has been employed in the study. So it is worthwhile to mention some of the works done on the line. Jagerman [12] discussed the time behavior of blocking in a fully available N-trunk group whose rate parameter itself was considered to vary with time. Rothkopf and Oren [21] studied effective computational methods for dealing with queues having non-stationary arrival processes. Heyman and Whitt [10] dealt the asymptotic behavior of the $\mathrm{M}_{\mathrm{t}} / \mathrm{G} / \mathrm{c}$ queue having a Poisson arrival process with a general deterministic intensity. Green et al. [9] studied a better understanding of how non-stationary affects delays in queueing systems. Green and Kolesar [8] discussed an easy - to - compute approximation for determining long run average performance measures for multi server Markovian queues with periodic arrival rates. Eick et al. [6] developed a better understanding of the time-dependent behavior in $\mathrm{M}_{\mathrm{t}} / \mathrm{G} / \infty$ queueing model. Dong and Whitt [5] investigated the consequences of fitting a birth and death (BD) process to a multi-server queue with a periodic time varying arrival rate function to better understanding of system.

In the present study, we give some insights into $M(t) / M / 1$ queueing model under the provision of sinusoidal arrival rate function and obtain various performance measures such as expected number of customers in the system, expected number of customers in the queue, expected time 
per customer in the system, expected waiting time per customer in the queue, traffic intensity. To show that our model studied is practically applicable we show the numerical results for various parameters change. Our model can be applied in the practical field where there is only one server and arrival of the customers in the system is periodic. It has ubiquitous applications in the world where the security has become the great issue.

\section{Mathematical Model}

The notations used for our model are as follows:

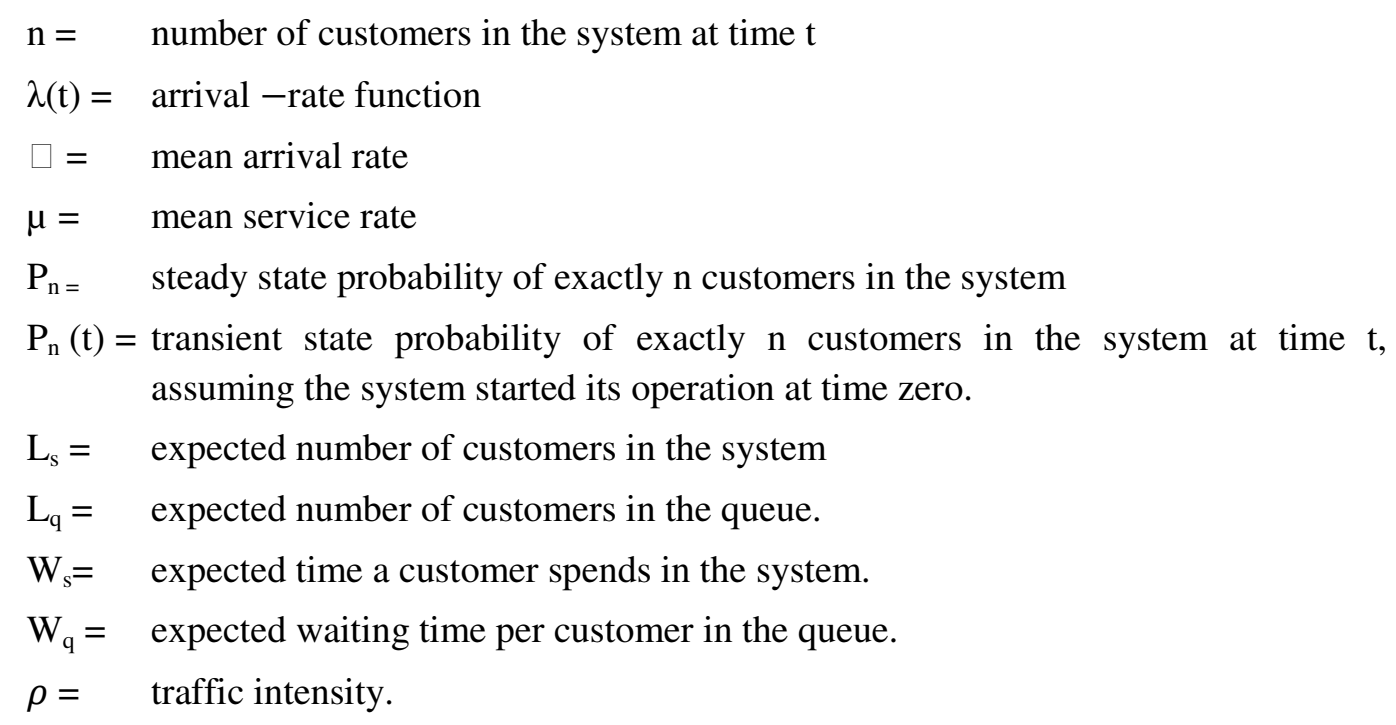

The transition diagram for our model is shown in figure 1.

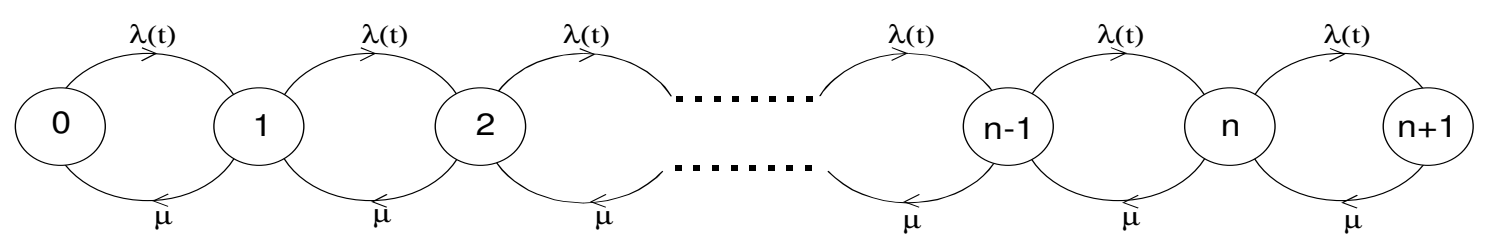

Fig. 1: Transition diagram

With the help of above transition diagram, the transition differential equations are:

$$
\begin{gathered}
P_{0}^{\mid}(t)=\mu P_{\mid}(t)-\lambda(t) P_{0}(t) \\
P_{n}^{\mid}(t)=\lambda(t) P_{n-1}(t)+\mu P_{n+1(t)}-[\lambda(t)+\mu] P_{n}(t), \text { where } n>0
\end{gathered}
$$

In steady state service system, when $t \rightarrow \infty, P_{0}^{1}(t) \rightarrow 0, P_{n}^{1}(t) \rightarrow 0$

$$
\begin{gathered}
\therefore \mu P_{1}-\lambda(t) P_{0}=0 \\
\lambda(t) P_{n-1}+\mu P_{n+1}-[\lambda(t)+\mu] P_{n}=0
\end{gathered}
$$

Solving (3) and (4) recursively, we have 


$$
P_{n}=\left[\frac{\lambda(t)}{\mu}\right]^{n} \cdot P_{0}, \quad n>0
$$

Now, next step is to find $\mathrm{P}_{0}$ by using normalizing condition

$\sum_{n=0}^{n} P_{n}=1$ that yields

$$
\begin{aligned}
P_{0} & =\frac{1}{\sum_{n=0}^{n}\left[\frac{\lambda(t)}{\mu}\right]^{n}} \\
\therefore P_{0} & =1-\frac{\lambda(t)}{\mu}
\end{aligned}
$$

Hence,

$$
P_{n}=\left[\frac{\lambda(t)}{\mu}\right]^{n}\left[1-\frac{\lambda(t)}{\mu}\right]
$$

\section{Performance measures of the system}

3.1 Expected number of customers in the system

$$
\begin{aligned}
L_{s}= & \sum_{n=0}^{\infty} n P_{n}=\sum_{n=0}^{\infty} n\left[\frac{\lambda(t)}{\mu}\right]^{n}\left[1-\frac{\lambda(t)}{\mu}\right] \\
= & {\left[1-\frac{\lambda(t)}{\mu}\right] \sum_{n=0}^{\infty} n\left[\frac{\lambda(t)}{\mu}\right]^{n} } \\
& =\left[1-\frac{\lambda(t)}{\mu}\right]\left[\frac{\lambda(t)}{\mu}+2\left(\frac{\lambda(t)}{\mu}\right)^{2}+3\left(\frac{\lambda(t)}{\mu}\right)^{3}+\cdots\right] \\
= & \left.11-\frac{\lambda(t)}{\mu}\right]\left[\frac{\frac{\lambda(t)}{\mu}}{\left(1-\frac{\lambda(t)}{\mu}\right)^{2}}\right]=\frac{\frac{\lambda(t)}{\mu}}{1-\frac{\lambda(t)}{\mu}} \quad \therefore L_{s}=\frac{\lambda(t)}{\mu-\lambda(t)}
\end{aligned}
$$

\subsection{Expected number of customers in the queue}

$\mathrm{L}_{\mathrm{q}}=$ expected number of customer in the system-expected number in service (single server)

$=L_{S}-\frac{\lambda(t)}{\mu}$

$=\frac{\lambda(t)}{\mu-\lambda(t)}-\frac{\lambda(t)}{\mu}$

$$
\therefore L_{q}=\frac{\lambda(t)}{\mu} \cdot \frac{\lambda(t)}{\mu-\lambda(t)}
$$

3.3 Expected time per customer in the system

$W_{s}=\frac{\text { expected number of customers in the system }}{\text { arrival rate }}$ 
$124 M(t) / M / 1$ Queueing System with Sinusoidal Arrival Rate

$$
\begin{gathered}
=\frac{L_{s}}{\lambda(t)}=\frac{\lambda(t)}{\mu-\lambda(t)} \cdot \frac{1}{\lambda(t)} \\
\therefore W_{s}=\frac{1}{\mu-\lambda(t)}
\end{gathered}
$$

3.4 Expected waiting time per customer in the queue

$$
\begin{gathered}
W_{q}=\text { expected time in the system }- \text { time in service } \\
=W_{s}-\frac{1}{\mu}=\frac{1}{\mu-\lambda(t)}-\frac{1}{\mu} \\
\therefore W_{q}=\frac{\lambda(t)}{\mu} \cdot \frac{1}{\mu-\lambda(t)}
\end{gathered}
$$

3.5 Traffic intensity

$$
\rho=\frac{\lambda(t)}{\mu}
$$

\section{Results and Discussion}
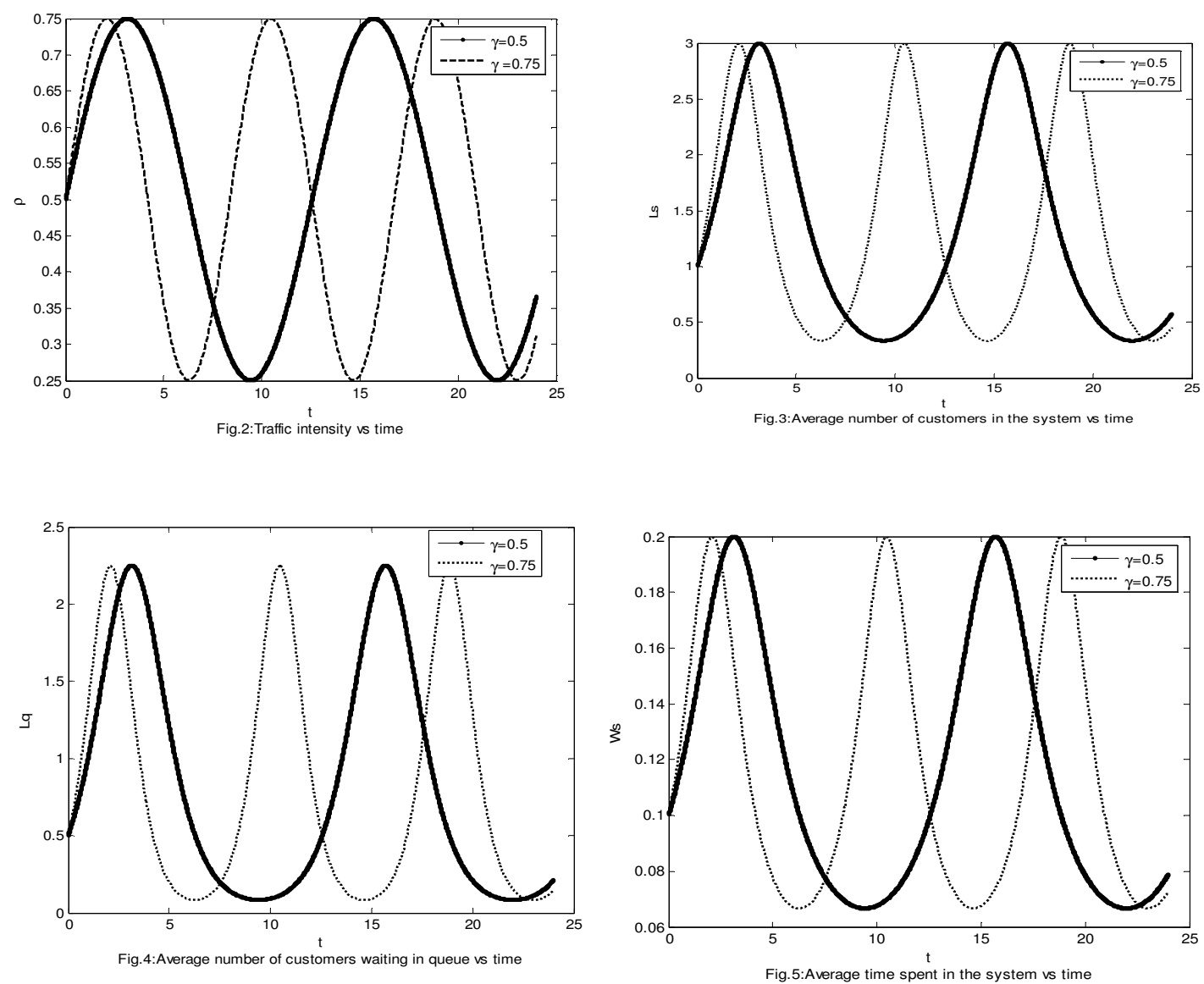
Pant and Ghimire 125
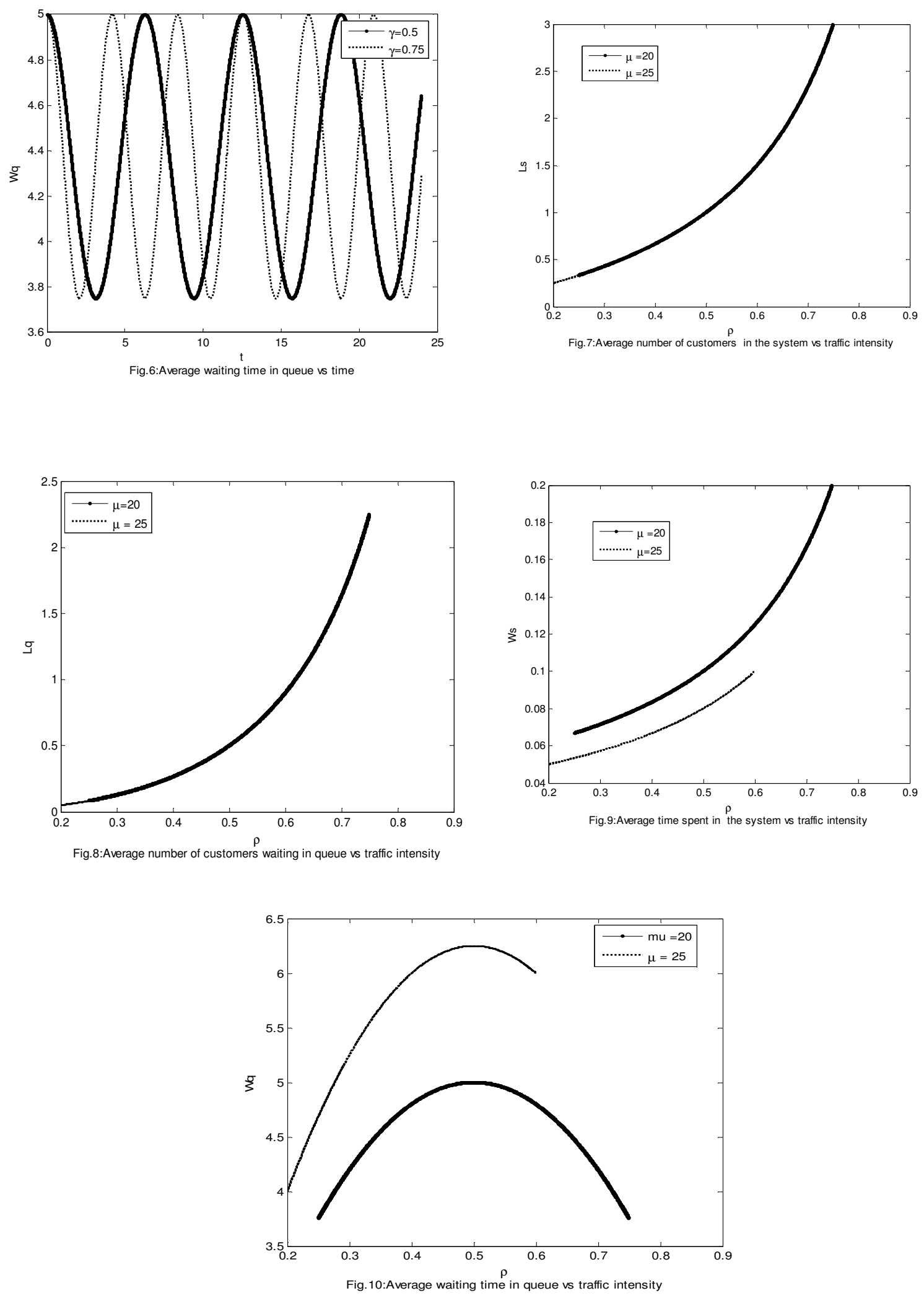
Fig. (2) Explores that smaller the value of relative frequency $\gamma$, the longer the time that the system take to achieve higher value of traffic intensity. This implies that longer the time to have customers in the queue. Fig. (3) predicts that smaller the value of $\gamma$, longer is the cycle time and slower rate to accumulate the customers in the system as well as in the queue. Fig. (4) displays that customers are waiting in queue from beginning. But as time passes on, customers in queue increases faster when $\gamma=0.75$ than $\gamma=0.5$, which is realistic. Fig. (5) shows that cycle time spent in the system increases faster when we take $\gamma=0.75$ than $\gamma=0.5$. This is also practical in real life situation. Fig. (6) explains that in the beginning of time, customers have already waited and as the time increase, waiting time decreases sharply for a while and again repeats the process. Cycle time is slower when $\gamma=0.5$ than $\gamma=0.75$. This can be experienced in the border crossing area in the check point etc. Fig. (7) shows that for fixed $\gamma$, initially the server is less busy when service rate is $\mu=25$ but when service rate decreases to $\mu=20$, the server is more busy. When numbers of customers in the system reach higher value, the traffic intensity increases fast, this is practically true. Fig.(8) predicts that when service rate is $\mu=25$, server is busy from beginning but when $\mu=20$, server is more busy after sometimes of the starting of the system. But for both value of $\mu$ waiting number of customers gradually increases simultaneously as the busy factor increases. Fig. (9) elaborates that lesser is the service rate, more is the time spent in the system. When service rate $=25$, customer has not to wait in the beginning. But afterwards they have to wait more than the customers when the service rate $=20$, which is inherently true. Fig. (10) shows that when service rate $=25$, customers have already waited in the queue but this is not so in the case when service rate is $\mu=20$. Waiting time is higher when service rate is higher. Lesser the service rate, below is the graph. Higher the service rate, above is the graph. But this is for a certain values of utilization factor in a cycle.

\section{Conclusion}

We have obtained various performance measures such as expected number of customers in the system, expected number of customers in the queue, expected time per customer in the system, expected waiting time per customer in the queue, traffic intensity. To show that our model studied is practically applicable we show the numerical results for various parameters change. Our model can be applied in the practical field where there is only one server and arrival of the customers in the system is periodic. It has ubiquitous applications in the world where the security has become the great issue. Such model was applied very well not only in modern age but also in the ancient days knowingly or unknowingly such as first come first served service discipline has been well implemented in RAMAYANA and MAHABHARAT. Our model can be studied as a further research under the provision of sinusoidal service rates with transient consideration.

\section{References}

[1] Abate J and Whitt W (1987), Transient Behavior of the M/M/1 Queue: Starting at the Origin. Queueing Systems 2: 41-65.

[2] Abate J and Whitt W (1988), Transient Behavior of the M/M/1 Queue via Laplace transforms. Applied probability Trust 20 : 145-178. 
[3] Baccelli F and Massey WA (1989), A Sample Path Analysis of the M/M/1 Queue. Journal of Applied Probability 26 (2) : 418-422.

[4] Choudhury A and Borthakur AC (2007), Statistical Inference in M/M/1 Queues: A Bayesian Approach. American J. of Mathematical \& Management Sciences 27(1-2):25-41.

[5] Dong J and Whitt W (2015), Stationary Birth- and- Death Processes Fit to Queues with Periodic Arrival Rate Functions.

www.columbia.edu/ ww 2040/periodic_BD_nrl_011715 ww.pdf: 1-24.

[6] Eick SG, Massey WA and Whitt W (1993), Mt/G/œ Queues with Sinusoidal Arrival Rates. Management Science 39 (2) : 241-252.

[7] Ghimire RP and Ghimire S (2011), Heterogeneous Arrival and Departure M/M/1 Queue with Vacation and Service Breakdown. Management Science and Engineering 5 (3): 1-7.

[8] Green L and Kolesar P (1991), The Point wise Stationary Approximation for Queues with Non-stationary Arrivals. Management Science 37 (1) : 84-97.

[9] Green L, Kolesar P and Svoronos A (1991), Some Effects of Non Stationarity on Multi server Markovian Queueing Systems. Operations Research 39 (3) : 502-511.

[10] Heyman DP and Whitt W (1984), The Asymptotic Behavior of Queues with Time-varying Arrival Rates. Journal of Applied Probability 21 : 143-156.

[11] Ibe OC and Isijola OA (2014), M/M/1 Multiple Vacation Queueing Systems with Differentiated Vacations. Modelling and Simulation in Engineering ID158247 : 1-6.

[12] Jagerman DL (1975), Non-Stationary Blocking in Telephone Traffic, Bell System Technical Journal 54 : 625-661.

[13] Jindal I and Sharma S (2012), A New Measure for M/M/1 Queueing System with Non Preemptive Service Priorities. International Journal of IT, Engineering and Applied Sciences Research 1(1) : 36-41.

[14] Kalidass K and Ramanath K (2014), Transient Analysis of an M/M/1 Queue with Multiple Vacations. Pakistan Journal of Statistics \& Operation Research X (1):121-130.

[15] Kinateder K K J and Lee E Y (2000), A new Approach to the busy period of the M/M/1 Queue. Queueing System 35: 105-115.

[16] Li H, Zhu Y, Yang P and Madhavapeddy S (1996), On M/M/1 Queues with a Smart machine. Queueing Systems 24: 23-36.

[17] Maurya V (2012), Investigation of Probability Generating Function in an Interdependent M/M/1: ( $\infty$, GD) Queueing Model with Controllable Arrival Rates using Rouche's Theorem. Open Journal of Optimization 1 (2) : 34-38.

[18] Maurya VN (2015), Mathematical Modelling and Steady State Performance Analysis of a Markovian Queue with Heterogenerous Servers and Working Vacation. American Journal of Theoretical and Applied Statistics 4 (2-1) : 1-10.

[19] Murthy TSR, Krishna DSR and Raju GVS (2012), M/M ${ }^{(\mathrm{k})} / 1$ Queueing model with varying Bulk service. Int. Journal of Mathematics and Soft Computing 2 (1): 109-117.

[20] Rasouli R, Ahmadi M and Ahmadvand A (2013), Energy Consumption Estimation in Clustered wireless Sensor Net works using M/M/1 Queuing model. International Journal of Wireless and Mobile Networks 5 (1) :15-31.

[21] Rothkopf MH and Oren SS (1979), A Closure Approximation for the Non-Stationary M/M/S Queue. Management Science 25 (6) : 522-534. 